\title{
Left bundle branch pacing in patients with right bundle branch block
}

\author{
Kailun Zhu',2, Yali Sun 1,2, Binni Cai', Linlin Li', Guiyang Li', Jianghai Liu', Xingcai Wan', Dong Chang', Qiang Li' \\ 'Department of Cardiology, Xiamen Cardiovascular Hospital, Xiamen University, Xiamen, China \\ ${ }^{2}$ School of Medicine, Xiamen University, Xiamen, China
}

\author{
Correspondence to: \\ Qiang Li, MD, \\ Department of Cardiology, \\ Xiamen Cardiovascular \\ Hospital, \\ Xiamen University, Xiamen, \\ Fujian 361000, China, \\ phone: +8615359293670 \\ e-mail: liqiang@xmu.edu.cn \\ Copyright by the Author(s), \\ 2021 \\ Kardiol Pol. 2021; \\ 79 (10): 1127-1129: \\ DOl: 10.33963/KP.a2021.0091 \\ Received: \\ May 26, 2021 \\ Revision accepted: \\ August 15, 2021 \\ Published online: \\ August 15, 2021
}

\begin{abstract}
INTRODUCTION
The paced morphology similar to right bundle branch block (RBBB) in lead V1, which usually featured Qr, qR, rSR', or QS patterns, is one of the expected parameters during left bundle branch pacing (LBBP) implantation [1-3]. It is due to the early activation of the left ventricle (LV) and delayed activation of the right ventricle (RV) (Figure 1A). A possible explanation for this phenomenon is that since LV excitation precedes RV excitation, then the paced QRS morphology of patients with RBBB produced by LBBP can be significantly different from the intrinsic one, especially that the paced QRS duration has been always narrower than the intrinsic duration. (Supplementary material, Figure S1A-S1D). We herein discuss several possible mechanisms to explain this shortening.
\end{abstract}

\section{METHODS}

This single-center prospective self-control study enrolled 32 patients who underwent LBBP, with complete right bundle branch block (CRBBB) but not incomplete or intermittent RBBB, and without other conduction disturbance (left posterior fascicular block, left anterior fascicular block, or septal fascicular block). The surface electrocardiograms (ECGs) with the preoperative diagnosis of CRBBB were carefully identified according to the criteria that included the QRS duration $>0.12 \mathrm{sec}$, a rSR' or RR' pattern in leads V1 and/or V2 and a wide and slurred $S$ wave in leads $V 6$ and I (S $>R$ duration or $S$ wave duration $\geq 0.06 \mathrm{sec}$ ). The process of LBBP has been previously described [1,2]. Both selective LBBP (SLBBP) and non-selective LBBP (NSLBBP) were acceptable. The definition of LBB capture was the constant Stim-LVAT (measured from the onset of the stimulus artifact to peak R-wave in lead V6), regardless of different outputs. We used the criteria proposed by Jastrzębski M et al. [4] to differentiate between LBBP and left ventricular septal pacing in patients with cRBBB. On each pair of ECGs, we measured both intrinsic QRS duration (iQRSd), from the onset to the end of the QRS, and paced QRS duration ( $\mathrm{pQRSd}$ ), following closely the pacing spike from the first deviation from baseline to the end of QRS. The local Ethics Committee approved the study protocol, and all of the patients provided their informed, voluntary, and written consent for participation.

\section{Statistical analysis}

The continuous variables were expressed as the means (SD), and the categorical variables were expressed as percentages. QRS duration before and after LBBP in each pair of subjects was compared by using paired-samples t-tests. Statistical analyses were performed with SPSS 22.0 (SPSS Inc, Armonk, NY, USA). $P$-values of less than 0.05 were considered to be statistically significant.

\section{RESULTS AND DISCUSSION}

The mean age of the participants was 72.76 (8.71) years, and there were 25 males in total (73.53\%). Three patients had sick sinus syndrome (SSS) whereas the rest of the participants had high-grade atrioventricular conduction blocks (AVBs). Postoperative ECGs were recorded under unipolar pacing configuration in 15 patients and under bipolar pacing configuration in the remaining patients. There was a significant decrease (compared to the iQRSd) with the pQRSd (144.31 [4.83] ms vs. 115.58 [5.80] $\mathrm{ms}$, respectively; $P<0.001)$, and the $\mathrm{pQRSd}$ 


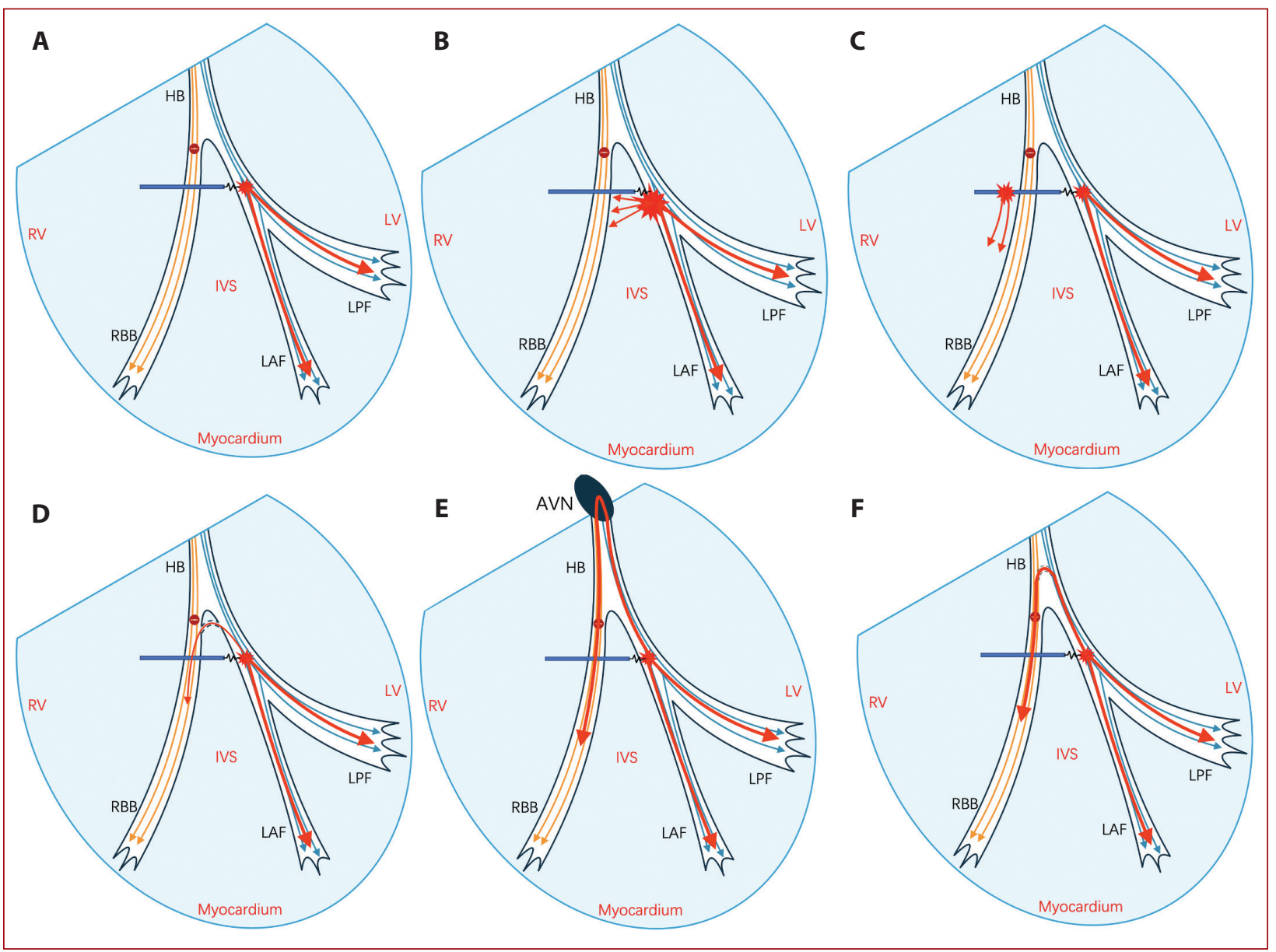

Figure 1. Schematic diagram of excitation conduction of left bundle branch pacing. A. Selective left bundle branch pacing (SLBBP) captures only the left bundle branch (LBB) to excite the left ventricle (LV) and then excites the right ventricle (RV) via the intercalated discs of the myocardium. B. Non-selective left bundle branch pacing (NSLBBP) causes fusion of excitation of the LV, the LBB area, and the local RV septum. C. Anodal capture occurred in the bipolar pacing configuration, wherein a portion of the RV septum was pre-excited by the anodal right ventricular ring. D. LBBP may bypass the right bundle branch (RBB) block through transverse interconnection fibers (TF)/functional transverse interconnection (FTI) (dashed line) and eventually activate RBB. E. The impulse is anterogradely conducted through the LBB to activate the $\mathrm{LV}$, after which it is retrogradely conducted to the atrioventricular node and then recruits the RBB. F. TF/FTI (dashed line) may also exist within His bundle $(\mathrm{HB})$, wherein it laterally connects to $L B B$ and RBB, which can transmit the retrograde impulse from $L B B$ to RBB and then capture RBB. See the text for details

Abbreviations: LPF, left posterior fascicular; LAF, left anterior fascicular; IVS, intraventricular septum

was 28.74 (3.30) ms shorter than the iQRSd. A similar shortening was observed in both unipolar (Figure 1B) and bipolar (Figure 1C) pacing configurations, as shown in Supplementary materials, Table S1.

The 3 possible mechanisms for these results are as follows.

\section{An excited fusion of the LV and local RV septum}

NSLBBP and anodal capture induce excited fusion of the LV and local septum. Some studies have described LBBP in a patient with RBBB, where the $\mathrm{R}^{\prime}$ wave peak time (measured from the onset of the stimulus artifact to peak $\mathrm{R}^{\prime}$-wave in lead V1) and $\mathrm{R}^{\prime}$ wave duration in the end of QRS complex were shorter in NSLBBP than in $\operatorname{SLBBP}[5,6]$. With the increase in pacing output, SLBBP is converted into NSLBBP with no isoelectric interval (Figure 1B), thus indicating that both LBB and local adjacent septal myocardium are captured.
The cathode tip of lead was placed in the LBB area (trunk or left anterior and posterior fascicle of the LBB), and the anode ring was located in the right ventricular septum. During a bipolar pacing configuration at high output, the anodal right ventricular ring can pre-excite a portion of the right septum to compensate for the RV delay (Figure 1C). This minimizes QRS duration because at least 2 depolarization wavefronts activate the ventricles, thus shortening the conduction time from the LV to the RV. The QRS morphology generated by anodal capture is the general shortened QS pattern without $\mathrm{R}$ wave on lead $\mathrm{V} 1$, which is significantly different from the Qr pattern generated by NSLBBP in the presence of short Stim-LVAT.

\section{Transverse interconnection between the LBB and the RBB}

The His-Purkinje system is complex, variable, and interconnected. In the 1970s, Lazzara et al. suggested that 
a functional transverse interconnection (FTI) exists in the His bundle (HB) and in the bundle branches (Figure 1F), which transmit impulses across the fibers [7]. Chu et al. [8] described LBBP as a possible treatment for correcting the RBBB through transverse interconnection fibers (TFs) that connect the LBB and RBB (Figure 1D). However, based on the properties of anisotropic conduction of longitudinal dissociation [7], it is suspected that the combination of the rapid longitudinal conduction of the impulse to the LV and the slow lateral conduction to the RV would result in a shortened $\mathrm{pQRSd}$. If transverse interconnection coexists with longitudinal dissociation, then the impulse generated by LBBP circumnavigates the structure block to excite the RV without a paced morphology of the RBBB. In addition, the anatomical structure of these fibers has not been previously reported.

\section{Retrograde conduction from $L B B$ to $R B B$}

It is clear that impulses can be bidirectionally transmitted in the His-Purkinje system. During LBBP, the impulse anterogradely captured the LBB to excite the LV and retrogradely captured the RBB to excite the RV [9]. In these circumstances, the pulse will retrogradely propagate in the LBB fibers to a turnaround point on its way up and create a deviation from the LBB to the RBB. Of course, the location of the turnaround point is important, wherein it is possibly located in the LBB and RBB (Figure 1D), in the proximity of $\mathrm{HB}$ (Figure 1F), and in the atrioventricular node (Figure 1E). However, even if the impulse can deflect from the LBB to the RBB, it is not clear whether it can recruit the distal RBBB.

In summary, excited fusion is most likely to shorten QRS duration after LBBP in patients with RBBB, and the specific mechanisms may be multifactorial, which requires more precise mapping and further anatomical study of the conduction system of the heart.

\section{Supplementary material}

Supplementary material is available at https://journals. viamedica.pl/kardiologia_polska.

\section{Article information}

Conflict of interest: None declared.

Open access: This article is available in open access under Creative Common Attribution-Non-Commercial-No Derivatives 4.0 International (CC BY-NC-ND 4.0) license, allowing to download articles and share them with others as long as they credit the authors and the publisher, but without permission to change them in any way or use them commercially. For commercial use, please contact the journal office at kardiologiapolska@ptkardio.pl.

How to cite: Zhu K, Sun Y, Cai B, et al. Left bundle branch pacing in patients with right bundle branch block. Kardiol Pol. 2021; 79(10): 1127-1129, doi: 10.33963/KP.a2021.0091.

\section{REFERENCES}

1. Huang W, Chen X, Su L, et al. A beginner's guide to permanent left bundle branch pacing. Heart Rhythm. 2019; 16(12): 1791-1796, doi: 10.1016/j. hrthm.2019.06.016, indexed in Pubmed: 31233818.

2. Sundaram S, Vijayaraman P. Left bundle branch pacing. Herzschrittmacherther Elektrophysiol. 2020; 31(2): 124-134, doi: 10.1007/s00399020-00694-8, indexed in Pubmed: 32458056.

3. Jastrzębski M, Moskal P. Reaching the left bundle branch pacing area within 36 heartbeats. Kardiol Pol. 2021; 79(5):587-588, doi: 10.33963/KP.15914, indexed in Pubmed: 34125940.

4. Jastrzębski M, Kiełbasa G, Curila K, et al. Physiology-based electrocardiographic criteria for left bundle branch capture. Heart Rhythm. 2021; 18(6): 935-943, doi: 10.1016/j.hrthm.2021.02.021, indexed in Pubmed: 33677102.

5. Gao MY, Tian Y, Shi L, et al. Electrocardiographic morphology during left bundle branch area pacing: Characteristics, underlying mechanisms, and clinical implications. Pacing Clin Electrophysiol. 2020; 43(3): 297-307, doi: 10.1111/pace.13884, indexed in Pubmed: 32045008.

6. Chen X, Wu S, Su L, et al. The characteristics of the electrocardiogram and the intracardiac electrogram in left bundle branch pacing. J Cardiovasc Electrophysiol. 2019; 30(7): 1096-1101, doi: 10.1111/jce.13956, indexed in Pubmed: 31094058.

7. Lazzara R, Yeh BK, Samet P. Functional transverse interconnections within the His bundle and the bundle branches. Circ Res. 1973; 32(4): 509-515, doi: 10.1161/01.res.32.4.509, indexed in Pubmed: 4702043.

8. Chu SY, Sheng QH, Chen ED, et al. Is the left bundle branch pacing a choice to conquer the right bundle branch block?-A case report. Ann Noninvasive Electrocardiol. 2021; 26(2): e12797, doi: 10.1111/anec.12797, indexed in Pubmed: 32896950.

9. Zhang J, Wang Z, Zu L, et al. Simplifying physiological left bundle branch area pacing using a new nine-partition method. Can J Cardiol. 2021; 37(2): 329-338, doi: 10.1016/j.cjca.2020.05.011, indexed in Pubmed: 32428620. 